\title{
"a perfectly natural thing to put it to music" - John Cage, Bernd Alois Zimmermann, Elisabeth Lutyens und Steve Reich komponieren Wittgenstein
}

\begin{abstract}
After I had played about ninety-eight exercises I was ready to do a little improvising. I used to take a fistful of chords and crash the piano from one end to the other, then sullenly modulate into "the Burning of Rome" or the "Ben Hur Chariot Race" which everybody liked because it was intelligible noise. Long before I read Wittgenstein's Tractatus Logico-Philosophicus I was composing the music to it, in the key of sassafras.

(Miller, [1939] 1961: 248) ${ }^{1}$
\end{abstract}

Die Vertonung genuin philosophischer Texte hat in der Musik Seltenheitswert. Zwar wurden Komponisten immer wieder durch philosophische Lehren beeinflusst, eine direkte Umsetzung philosophischer Texte scheint jedoch in den allermeisten Fällen kaum denkbar. Umso erstaunlicher ist die schiere Anzahl der Kompositionen, die auf Wittgensteins Texten fußen. Der britische Komponist Anthony Powers, dessen Arbeit A Picture of the World (für Countertenor, Klarinette und 16-stimmigen Chor, 2001) genau dies unternimmt, behauptet gar: „The phrasing, the form of words he uses, the way some sentences look as if they have meaning but actually go nowhere, are all amazingly musical, and it seems a perfectly natural thing to put it to music.“ (Tait, 2003: o.s.) Beziehungen der Wittgenstein'schen Sprache zur Musik wurden immer wieder hergestellt, bereits einer der ersten Tractatus-Interpreten verwies auf die musikalische Grundstruktur des Werkes:

\footnotetext{
Wenn man die Nummerierung [des Tractatus] als ein Gegenstück zu den Zeichen ansieht, mit denen in Notenschrift die Variation der Tonstärke bezeichnet wird, bekommt man eine Vorstellung von dem wogenden Rhythmus des Traktats und seiner Einteilung in Haupt- und Nebenthemen. (Stenius, 1969: 17) ${ }^{2}$
}

1 Der Sassafras-Baum bildet den Rohstoff Safrol zur Herstellung von Root-Beer, der Droge Ecstasy sowie mehrerer Psychopharmaka.

2 Stenius geht es vor allem um den Rhythmus: „[...] man kommt der Sache tatsächlich am nächsten, 
Toru Takemitsu ließ sich 1969 für sein Werk Stanza I (für Gitarre, Klavier, Celesta, Harfe, Vibraphon und Sopran) von dem Tractatus-Satz 6.432 inspirieren: „Wie die Welt ist, ist für das Höhere vollkommen gleichgültig. “ Laurie Anderson widmete Wittgenstein zwei Songs über dessen Sätze „If You Can't Talk About it, Point to it“ und „Language is a Virus from Outer Space“, und Donnacha Dennehy komponierte 2000 Counting (für Streichquartett und Elektronik). Ein Beispiel aus jüngerer Zeit ist Balduin Sulzers Tractatus logico-philosophicus (für Sopran solo und unterschiedliche Klanggeräte, 2007). Bekannt ist auch die Tractatus-Suite des finnischen Künstlers Mauri Antero Numminen aus ausgewählten Sätzen des Tractatus von 1966, bestehend aus sechs Liedern verschiedener Stile bzw. Gattungen wie Jazz, Rock oder Walzer, einem atonalen Lied sowie dem Marsch Wovon man nicht sprechen kann. Numminens absurd verstellte Stimme singt diesen Satz in deutscher Sprache mehrfach abwechselnd mit martialisch anmutendem Männerchor, unterlegt von absichtlich dilettantischer Marschmusik, und konterkariert damit in Kurt Weill'scher Bissigkeit eine allzu oft zur leeren Phrase abgeflachte Verwendung dieses bekannten Satzes.

Wie es sich mit dieser so „natürlichen Sache“ wie es Anthony Powers nennt, Wittgenstein $\mathrm{zu}$ vertonen, tatsächlich in einigen Fällen verhalten hat, möchte ich anhand von vier Komponisten und Werken näher betrachten. Diese reizte an Wittgenstein anscheinend (mindestens) drei verschiedene Dinge zu einer musikalischen Darstellung: die Idee des Sprachspiels (hierzu John Cage und Bernd Alois Zimmermann), die Struktur seiner Schriften (hierzu Elisabeth Lutyens) und die „Idee einer Idee“ selbst (Steve Reich).

\section{Musikalisierte Sprachspiele: Cages I-VI (1988/89) und Zimmermanns Requiem für einen jungen Dichter (1967/69)}

John Cage widmete sich Wittgenstein in seinen „Harvard-Lectures“ (Charles Eliot Norton Lectures) 1988/89. Sie bestanden aus der an sechs lectures von Cage als Sprecher selbst aufgeführten Arbeit I-VI mit jeweils einer nachfolgenden Fragestunde für die Teilnehmenden. Cages Arbeit besteht aus auf etwa 400 Textseiten

wenn man sagt, die Nummerierung zeige eine Art Rhythmus des Nachdrucks, der Betonung. Die Sätze 1, 2, 3, 4, 5, 6, 7 können als „forte“-Stellen angesehen werden, denen natürlicherweise Decrescendos folgen - aber denen auch Crescendos vorausgegangen sind.“(ebd.). 
abgedruckten sogenannten „mesostics“, seiner bevorzugten kompositions-poetischen Form. Bilden bei einem Akrostichon Anfangs oder Endbuchstaben der horizontalen Zeilen vertikal gelesen einen neuen Begriff, steht der vertikal zu lesende Begriff bei einem mesostic in der Mitte der Zeilen, Cage hebt sie durch Großbuchstaben heraus. Unterhalb dieser mesostics läuft der ohne sprachliche Korrekturen transkribierte Text der Fragen des Auditoriums mit Cages Antworten in Kleinbuchstaben ohne jegliches Satzzeichen als „Kontrapunkt“ in der abgedruckten Version mit. Cages Antworten sind jeweils kursiv, die anonym bleibenden Fragen gerade gedruckt. Die entstehenden mesostics, die nacheinander abgehandelt werden, bilden den eigentlichen Titel der Arbeit: „Method, Structure, Intention, Discipline, Notation, Indeterminacy, Interpenetration, Imitation, Devotion, Circumstances, Variable Structure, Nonunderstanding, Contingency, Inconsistency, Performance.“ Als Materialbasis dienen ihm einzelne Zitate von Wittgenstein, Thoreau, Emerson, McLuhan und anderen, aber auch der New York Times, dem Wall Street Journal und dem Christian Science Monitor. Die Originaltexte sind im Anhang für jedes der mesostics aufgelistet. Mit einem Computerprogramm welches auf Operationen des I Ging beruht, reihen sich Bruchstücke aus den Zitaten auf der Basis von „chance operations“ aneinander. (Cage hatte bereits zuvor solche Zufallsoperationen für die Musik maßgeblich nutzbar gemacht und verwendet sie in vielen seiner Werke ausgiebig (Vergl. z. B. Perloff 1997).)

Wittgenstein nimmt dabei, Cages' eigener Einführung zufolge, eine prominente Rolle ein:

I have long been attracted to his work, reading it with enjoyment but rarely with understanding. Peter Yates introduced me to it. John Holzaepfel, who has written a text ${ }^{3}$ relating Wittgenstein's 'use' to my 'process', offered to help me by finding Wittgenstein's quotations suitable for some of my files. I accepted his help but found his choices as mysterious as the books from which they were taken. I decided to subject the Wittgenstein corpus to chance operations. Which book? which page? were my questions. Given the page I made a choice. Ninety-three entries were made in the fifteen files having nothing to do with the file names as subjects, unless by coincidence. (Cage 1990: 3)

Cage wählt aus sehr unterschiedlichen Werken Wittgensteins Passagen aus, darunter die Lectures 1932-1935, Lectures and Conversations, Philosophical Grammar, Culture and Value, Remarks on the Foundation of Mathematics oder dem Tractatus. Da es sich bei den Quelltexten - bis auf den Tractatus - bekanntlich nicht um originale Zusammenstellungen von Wittgenstein handelt, ist Cages Herangehensweise auf eine eigene Weise sehr konsequent: Er findet die Auswahl

3 Dieser Text wurde nie veröffentlicht. 
Holzaepfels nicht weniger „mysterious“ als die Texte selbst und tritt dann durch eine völlig idiosynkratrische Auswahl mit Unterstützung des Zufallsverfahrens von einer semantischen Auslegung Wittgensteins zurück: Er macht den Text zum reinen Klangmaterial, lässt ihn „geschehen“ und macht diese Performativität zu seiner eigenen ,Sprachkritik‘:

In the nature of the use of chance operations is the belief that all answers answer all questions. The nonhomogeneity that characterizes the source material of these lectures suggests that anything says what you have to say, that meaning is in the breath, that without thinking we can tell what is being said without understanding it. (Cage 1990: 6)

it is the same with a mathematical problem Do man's intErnal iT will opEn in a closed system ' such

one gives a child 'e.g. foR which it gets an answer according to the ' the world is our Mothertongue that varIables did Not the sAme ' result in

no vision ' etCetera ' no one will emptY

broken down that Is

Nor

every Day

spacE

know whaT

and honEstly clad but vaRiables in May be

by whIch

the Next room it might be or A gap like

those to which the

this would be approximatelY the area japan uses to feed her people ' the world Is our down that is

with a great pleasure and often and you can do it either in ordinary circumstances or in extraordinary circumstances it works very well in that walk through the woods on wednesday night one of the things that struck me was a lot of little rings of mushrooms not just single mushrooms but that little clusters of ideas that would sort of crop up and then disappear throughout the lecture and $i$ was wondering how much the

Abb.1: John Cage 1988/89: 27 (Ausschnitt)

In diesem Ausschnitt aus $I-V I$ ist die erste Zeile mit einiger Sicherheit einem Wittgensteinzitat entnommen, das „et cetera“ vielleicht ebenfalls, doch die Spur verliert sich, der Leser gerät ins Spekulieren, während sich der Hörer längst dem Fluss der Klänge anvertraut hat. Diese Herangehensweise ist in gewissem 
Sinne eine sehr konsequente Umsetzung Wittgenstein'scher Sätze, ein tatsächlicher „use“, wie Cage formuliert. Dem festesten Prinzip, dem Zufall überlassen, kann sich ein solcher Text selbst auslegen ohne feste Bedeutungen herzustellen. Sagen als klingendes und verschweigendes Sagen - es spricht nicht mehr der Text, sondern die Worte werden zu musikalischen Elementen, an denen nur noch Klang, Dauer, Rhythmus oder Tonhöhe aufgefunden werden können, die gleichzeitig aber ihre Bedeutungsgeschichte nicht verleugnen, sondern dieser sprachspielartig durch die Neu-Kontextuierung auf ungeahnte Weise erweitern. Dass Cage genau aus diesem Verständnis heraus arbeitet, dafür spricht seine Antwort aus dem „Kontrapunkt“ der Arbeit:

\begin{abstract}
i have the feeling as i work on these lectures you know from my introduction that i don't understand wittgenstein but nevertheless i'm using him i have the feeling that i'm beginning to understand him now at least more than i did and i told that to my friend $\mathrm{mr}$ holzaepfel who does understand his work and he said oh good and the $i$ told him what $i$ thought $i$ understood he sees more possibilities or more uses of things than just a few uses and ultimatively there are almost limitless uses then mr holzaepfel spoke of wittgenstein as someone who was able to ask questions that haven't been asked this had to do with creativity that is to say bringing into existence the things that hadn't been or hadn't been noticed so that you could then pay attention differently to other things (Cage 1990: 38-40).
\end{abstract}

Bernd Alois Zimmermann übernahm Zitate Wittgensteins in die Textmontagen des Prologs zu seinem Requiem für einen jungen Dichter (1967/69). Zwar ist bekannt, dass Zimmermann Teile von Wittgensteins Werk kannte, ob es sich jedoch um ein vertieftes Studium der sprachphilosophischen Ansichten Wittgensteins oder um ein Zusammentreffen ähnlicher Ansichten handelte, kann nicht genau rekonstruiert werden. (Vgl. Hiekel 1995: 65)

Ohne Frage jedoch bekommt der Sprachspiel-Begriff im Requiem eine programmatische Funktion:

Sprache wird [...] vor allem in ihrem sprachpragmatischen Aspekt, in der Vielfalt ihrer funktionalen Verwendungsweisen eingearbeitet. [...] Für Zimmermann ergibt sich dadurch die Möglichkeit, durch ein Auseinanderfallenlassen von semantisch-genetischem Gehalt der verwendeten Materialien und ihrer pragmatischen (Sprachspiel-)Funktion Spannungsverhältnisse zu komponieren, die politische, philosophische und religiöse Bedeutungsgehalte aufleuchten lassen. (Birnbacher 2008: 49)

Seine Zusammenstellung dieser „genetisch“ so unterschiedlichen Texte wie Passagen des Grundgesetzes, von Messtexten oder Zitaten aus dem Roten Buch Mao Zedongs, von James Joyce, Kurt Schwitters und Augustinus, werden mit akustisch vorfindlichem Material wie Originaleinspielungen von Hitler und Papst Johannes XXIII zusammengestellt. Die musikalische Zitatebene ist dabei ebenso komplex 
angelegt: Ausschnitte aus Wagners Tristan wechseln mit Messiaens L’Ascension, Beethovens Neunter Symphonie oder Hey Jude von den Beatles. Der Hörer muss so aktiv in die - ganz Wittgenstein'sche - „Erforschung von immer neuen Sinnzusammenhängen“ eintreten, und den wie Sprachspielkonfigurationen zusammengesetzten Deutungselementen im Gebrauch (hier: im Erklingen) neuen Sinn verleihen. (Vgl. Hiekel 1995: 634) Zimmermann hinterfragt durch solche Neukonfigurationen jedoch nicht nur die Ebene von Text und Musik-Zitaten, also vorfindlichem semantisiertem Material, sondern auch die historische Dimension von Musik selbst: Nicht nur werden traditionell festgeschriebene kompositorische Strategien mit neuen Herangehensweisen kombiniert und so neu definiert, sondern er tritt auch mit der Gattung des Requiems und ihrem historischen Kontext immer neu ins Gespräch.

Dass Wittgenstein nicht nur mit der Idee des Sprachspiels, sondern auch auf der konkreten Textebene eine besondere Rolle einnimmt zeigt sich zum Einen daran, dass es sich, auf etwa elf Minuten gedehnt, um das längste Textzitat der gesamten Komposition handelt. Außerdem stellt Zimmermann mit einer Bemerkung in der Partitur sicher, dass die Verständlichkeit dieser Textebene nicht durch andere musikalische Schichten beeinflusst werden darf. Mit seinen Textmontagen stellt Zimmermann immer wieder neu kombinierte semantische Situationen her, ähnlich wie Wittgenstein durch die immer wieder neue Zusammenstellung - allerdings nacheinander erscheinender thematischer Felder - neue „Belichtungen“ schafft. In gewissem Sinne führt Wittgenstein auf ähnlich „musikalische“ Weise in seinen Texten nicht nur immer neue Themen ein, variiert oder kontrastiert sie und führt sie in neue Kontexte, sondern man könnte für diese Texte sogar die Metapher einer sinfonischen Partitur bemühen: Wittgensteins multiperspektivisches Sprechen ist polyphon. Die verschiedenen Stimmen mit ihren individuellen Klangfärbungen erhalten eigene Rollen in der thematischen Arbeit, treten auf, verschwinden wieder, kommen später zusammen mit anderen in neuen Kontexten und neuen Verbindungen. ${ }^{5}$

\footnotetext{
4 Hiekel ist vor allem an Zimmermann interessiert, seine Ausführungen zu Wittgenstein sind daher recht kurz.

5 Einen sehr interessanten Versuch der optischen Darstellung dieser Polyphonie in Wittgensteins Texten hat Ingolf Max unternommen (Max 2010).
} 


\section{Musikalisierte Struktur: Elisabeth Lutyens Motet (1953)}

William Glock, Redakteur bei der BBC, der sich als Multiplikator um die zeitgenössische Musik in England verdient machte, war Freund und Förderer des enfant terribles Elisabeth Lutyens. Diese ist vor allem als eine der wenigen Vertreterinnen der Dodekaphonie in England bekannt, ihre Kompositionen sind durch Anton Webern angeregt, jedoch durchaus eigenständig. Sie hatte zudem eine enge Beziehung zu Strawinsky, der in den 50er Jahren ebenfalls zwölftönig komponierte. Glock gab bei Lutyens eine Vokalkomposition in Auftrag und lobte das so entstandene Werk später als ,a kind of geometry answering to Wittgenstein's philosophical thought.“ (Glock 1983: o. S.) Lutyens Motette Excerpta Tractatuslogico-philosophicus besteht aus 29 Sätzen des Tractatus, die kurzen Sätze übernimmt sie ganz, längere werden nur in Auszügen präsentiert, eher logische Komponenten von Sätze fallen gelegentlich zu Gunsten einer stärkeren Aussagewirkung weg (z. B. Takt 19-21 vertont Satz 2.021 „Die Gegenstände bilden die Substanz der Welt.“ Dabei fehlt der anschließende Teil „Darum können sie nicht zusammengesetzt sein.“) Zweimal wiederholt sie, vermutlich aus klanglichen wie inhaltlichen Gründen den Nachsatz „sind die Welt“ (Takt 38+44), und fügt in Takt 47 eigenmächtig „ein Bild der Welt“ hinzu, später „dass es sich so verhält“ (T 66f, „verhält“ hat eine gewisse klangliche Ähnlichkeit zu „Welt“).

Obwohl ihre Biographie (und in der Folge verschiedene Programmnotizen) behauptet, sie habe die durch Wittgensteins Nummerierung hervorgehobenen Propositionen vertont, ,which effectively constitued a summary of the thought of the book“ (so die Behauptung), ist das nicht der Fall: Die Hauptsätze 5 und 6 zur Wahrheitsfunktion fehlen, allerdings wäre der 6. Satz mit seiner enthaltenen Formel auch kaum hörerfreundlich vertonbar gewesen. Sie geht teilweise bis in die vierte Dezimalstelle der Tractatus-Nummerierung und wählt ohne erkennbares System vor allem (klanglich) starke Sätze zu den Themen Welt, Wirklichkeit, Wahrheit, Leben und Tod.

Im Gegensatz zu Cage, Reich (s.u.) oder Zimmermann beruhte dabei die Wahl des Textes nicht auf einer bereits früheren persönlichen Beschäftigung mit dem Philosophen. Auf der Suche nach einem Text, der keine jugendliche Liebeslyrik sein sollte, sondern ,something accurate and impersonal but not religios“ (Lutyens 1972: 222), stand offenbar zunächst Euklid zur Auswahl, Lutyens entschied sich erst auf Vorschlag ihres Freundes Terence Tiller für den Tractatus, von Wittgenstein hatte sie nach eigener Aussage bis $\mathrm{zu}$ diesem Zeitpunkt noch nie gehört. (Lutyens 1972: 223) Auch der kompositorische Prozess scheint zunächst nicht vornehmlich inhaltlich, sondern strukturell und klanglich orien- 
tiert gewesen zu sein: „From the first glance I knew I had found what I wanted, the words and ideas being ideally suited to the already formulated sound and architecture of the music in my mind.“ (Lutyens 1972: 223) Trotz der deutschenglischen Ausgabe war die Komponistin überzeugt, dass nur der Klang der deutschen Sprache ein Äquivalent zur musikalischen Architektur bilden könne, Ein Wort wie „Satz“ sei so einmalig in Klang und Bedeutung, dass die englische Übersetzung „proposition“ das Ergebnis nur stören könne. (Lutyens 1972: 223) Also ließ sich Lutyens von der befreundeten Komponistin Chula Doniach die deutschen Worte in musikalische Notation fassen und so lange in deren Klang unterrichten „until she knew the meaning of every sound.“ (Harris 1989: 157) Erst dann verband sie die bereits fertiggestellte Musik Silbe für Silbe mit den deutschen Worten (Harris 1989: 158).

Die Komposition für unbegleiteten Chor, op. 27, uraufgeführt 1953, ist mit „Motet“ überschrieben und folgt dem Modell der Gattung: Die Textpassagen werden klar hörbar nacheinander musikalisch „abgehandelt“, es wechseln homophon-syllabische Teile und kontrapunktische, z. T. kanonische, z.T. konzertierende Passagen einander ab. Das ganze Werk beruht auf einer Zwölftonreihe, die, ähnlich wie bei Werken Anton Weberns, aus zwei intervallisch symmetrischen Sechstonhälften gebaut ist, der zweite Teil ist einen Ganzton tiefer transponiert und erscheint als Krebs.

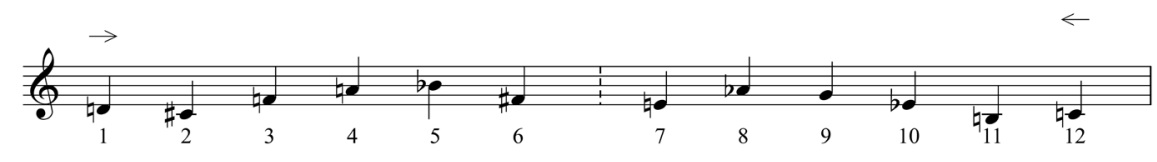

Abb. 2: Originalreihe für „Motet“

Der Schönberg'schen Reihenidee folgend lassen sich von dieser Reihe als Original, Krebs, Umkehrung und Krebsumkehrung 47 weitere Reihen ableiten (in diesem Falle sind sich, auf Grund der symmetrischen Reihengestalt, Originalgestalt und Krebs sehr ähnlich), die das gesamte Tonmaterial der Komposition bilden - in den polyphonen Teilen der Komposition horizontal, bei den homophonen Teilen vertikal als Akkordstrukturen verwendet. Beginn und Ende der Reihen korrelieren zumeist mit den vertonten Sätzen, bei längeren Sätzen lassen sich - dabei nicht nur linear sondern auch quer durch die Stimmen - mehrere Reihen auffinden. Durch die Intervallstruktur der Reihe können vier übermäßige Dreiklänge gebildet werden, mit denen Lutyens in der Vertikalen arbeitet. Sichtbar wird dies z. B. in den Takten 7-10. In Takt 7 erklingen alle 12 Töne, Lutyens schiebt jedoch die entstehenden übermäßigen Dreiklänge (die nie rein, sondern 
immer mit einem weiteren Ton gemeinsam erklingen) vertikal verschoben weiter. So bildet schließlich z. B. der Akkord in Takt 10 über „Welt“ die harmonische ,Essenz' der Reihe ab: zwei chromatisch übereinander geschichtete übermäßige Dreiklänge, die Bestandteil einer jeden Reihenhälfte sind, die übrigen Reihen-

\section{Motet}

Text taken from

Tractatus Logico-Philosophicus

Elisabeth Lutyens

Ludwig Wittgenstein

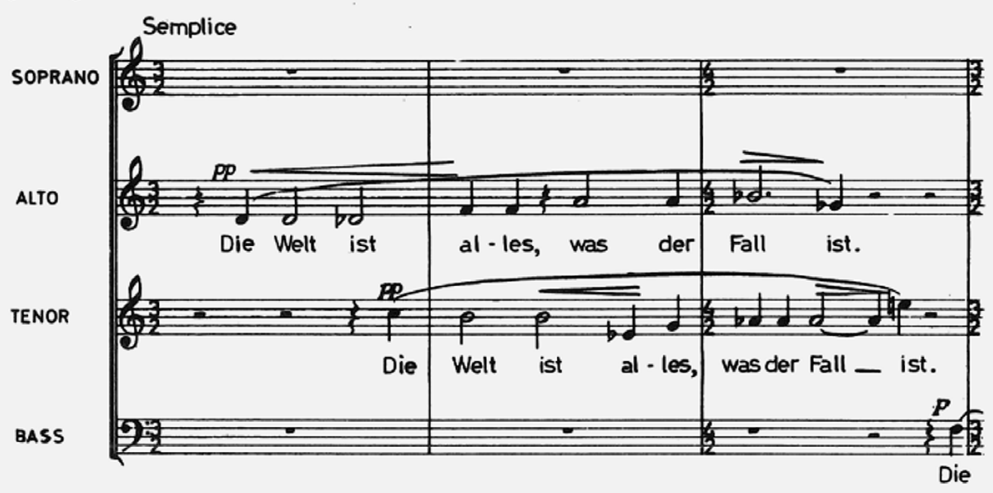

s.

B.

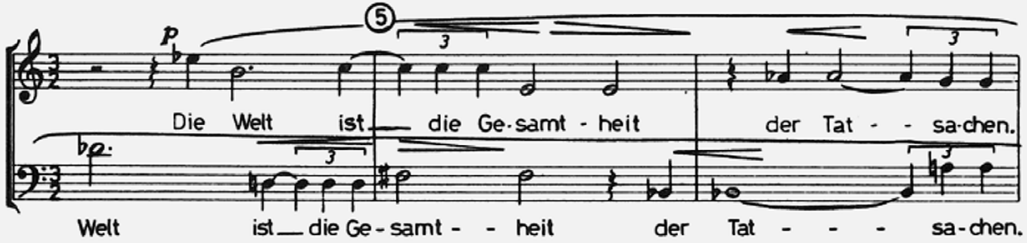

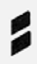

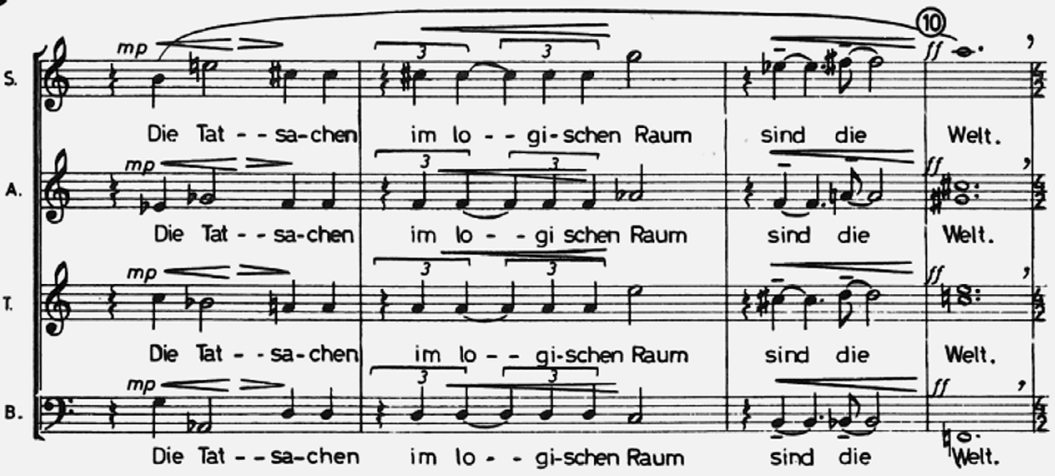

Abb. 3: Beginn von Motet (Lutyens, 1975: 1) 
töne finden sich im Takt davor. Das deutet auf eine bestimmte Interpretation des überzufällig häufig erscheinenden Wortes „Welt“ hin: in diesen beiden Takten werden alle vier übermäßigen Dreiklänge präsentiert, sie sind die „Tatsachen“, die im „logischen Raum“ unseres temperierten, westlichen Tonsystems tatsächlich die ganze „Welt“ sind, da dieses Tonsystem nur eben diese vier möglichen übermäßigen Dreiklänge kennt.

Die Ausgangsreihe findet sich neben dem Beginn des Stückes besonders auffällig noch an zwei prägnanten Stellen in (fast) originaler Gestalt: Am Schluss, in Terzen geführt von Sopran und Bass sowie an der auch klanglich hervorstechenden Solopassage in der Nähe des goldenen Schnitts (Takt 96-109), die mit Erreichen der Spitzentöne im Sopran das Thema ,Tod‘ in expressiver Weise abhandelt (in dieser Passage ändert sie auch zugunsten einer klareren Aussage den Text ab, T 106/7). Der Hauptaugenmerk Lutyens‘ liegt also auf einer sehr eigenenständigen und emotionalen Interpretation des Tractatus. Als korrespondierendes Element könnte man lediglich die logische Struktur begreifen: Wie im Tractatus die Propositionen von sieben Hauptsätzen ,abgeleitet‘ werden, und in einem bis heute diskutierten, und keineswegs sofort verständlichen Nacheinander angeordnet sind, leitet Lutyens ihr gesamtes Material von einer Tonreihe ab und bringt deren - sozusagen klanglich sinnvolle Sätze - in geordnetem, ebenfalls klanglich sinnvollen Nacheinander zur Erscheinung.

\section{Musikalisierte Idee: Steve Reich Proverb (1995)}

„How small a thought it takes to fill a whole life“ - dieser Satz aus der Kompilation Culture and Value liegt dem etwa 14 Minuten dauernden Stück Proverb (für drei Soprane, zwei Tenöre, zwei Vibraphone und vier MIDI Keyboards mit zwei Spielern) zugrunde, welches 1995 beim Early Music Festival Utrecht vom Theatre of Voices unter Paul Hillier uraufgeführt wurde.

Die offensichtlichste Beziehung Reichs zu diesem Satz ist schnell hergestellt ${ }^{6}$ : Als einer der Hauptvertreter der sogenannten „minimal music“ könnte er auch als Motto über dem kompositorischen Lebenswerk Reichs stehen, deren Ausgangspunkt oder Idee fast immer aus einem solchen „small thought“ besteht. Aber die Beziehung zwischen Reich und Wittgenstein geht tiefer und reicht weiter

$6 \mathrm{Zu}$ Proverb gibt es einen sehr instruktiven Aufsatz von Ronald Woodley, der auch auf die Wittgensteinbezüge eingeht (Woodley 2007). 
zurück. So sollte The Desert Music (1984) ursprünglich ebenfalls ein WittgensteinText zugrunde liegen und Reichs vielleicht bekanntestes Werk, Different Trains (1988), sollte sogar Wittgensteins gesampelte Stimme enthalten, was Reich offenbar nur aufgab, weil er keine Aufnahme von Wittgensteins Stimme finden konnte. (Woodley 2007: 465) Diese Faszination begann bereits in Reichs Philosophiestudium (1953-57) an der Cornell Universität, welches er mit einer Arbeit über Wittgensteins Spätwerk abschloss; in eigenen Bemerkungen zu Proverb erinnert der Komponist explizit daran:

\footnotetext{
As an undergraduate, what originally drew me to Wittgenstein was his idea that philosophical problems could be understood by looking at how we normally use language. For instance, the philosophical question, 'How can a mind (or soul) be inside a material body?' is using the noun 'mind' or 'soul' as 'spoon' or 'stone'. Wittgenstein asks how we would teach a word like 'mind' or 'soul' to a child. In what language games would we use these words? This kind of close, subtle examination of everyday speech had a strong appeal to me. As to his text that I used in Proverb, I was trying to embody it in the piece. That is, the 'small thought' is the idea of canon or round. (Reich 2002: 193)
}

Reich erforscht in Proverb diesen „kleinen Gedanken“ in vielerlei Hinsicht. Zuerst ist ein Kanon eine strenge musikalische Regel und kann nur gelingen, wenn dieser Regel gefolgt wird. Zusätzlich zum einfachen Kanon wendet Reich das Prinzip des Proportionskanons (nach franco-flämischem Vorbild) an. Beide Verfahren nutzend, spielt er dabei alle kompositorischen Grenzen dieser Idee durch: Wo man fast meditative Strenge regelkonformen Aufeinanderfolgens zu hören meint, breiten sich tatsächlich feine Risse in der kontrapunktischen und augmentierten Struktur der Komposition aus: die Mikrostrukturen als solche werden in ihrem harmonischen und rhythmische Potential ,vor Ohren' geführt und so entfaltet der „small thought“ sich im Verlauf der Komposition zu einem Spannungsfeld zwischen strengem Regelfolgen und Regelbruch: Wann ist eine Regel überhaupt noch als solche wahrnehmbar? Wann ist ein Kanon noch ein Kanon?

Die Idee der Augmentation leitet sich dabei bereits aus dem Thema ab, das selbst in sich augmentiert ist. Das Thema konstituiert durch seine Intervalle und seinen halbschlüssigen Charakter aber auch die Basis für Reichs andere klingende Untersuchung von sich entfaltenden Kanonregeln: er bezieht nicht nur Ideen der Renaissancemusik mit ein, sondern auch die Geschichte der mehrstimmigen Musik selbst wird „thematisiert“. Deutlich hörbar bezieht er sich auf Perotins Organa, die ältesten überlieferten vierstimmigen Musiken des Abendlandes. Hier werden die beiden ersten Worte des lateinischen Graduale über mehrere Minuten ausgekostet, Silbe für Silbe lösen sich so für heutige Ohren zu einem reinen Klangerlebnis auf. Perotin vertonte den liturgischen Text nicht mehr auf Verständlichkeit hin, sondern organisierte musikalisch-architektonische Merkmale, wie zum Beispiel Stimmentausch, über sehr kurze wie über längere Zeiträume hinweg. 
Das melismatische Singen über einer Silbe greift Reich auf, macht Anleihen bei der mittelalterlichen Modalrhythmik und übernimmt das enge Stimmengeflecht mit seinen Lagenwechseln (die Samples verstärken diesen Eindruck). Das ist zwar für eine analytische Betrachtung oder geübte Sänger erkennbar, doch selbst ein erfahrener Hörer nimmt in erster Linie die Wiederholungsstruktur selbst wahr: Eine Art Spiel zwischen Vorder- und Hintergrundstruktur entsteht. ${ }^{7}$ Unterstützt wird dieser Eindruck durch die Interpreten der Einspielung: Die bekannteste (und 1995 wahrscheinlich immer noch einzige) Aufnahme der Perotin'schen Organa stammt ebenfalls von den Sängern unter Paul Hillier, die klanglichen Ähnlichkeiten sind unüberhörbar. Reich kann also für seine „historische Kanonforschung“ zusätzlich zur musikalischen Faktur auch auf die Kategorie „Sound“ zurückgreifen. Reichs musikalisches „Nachdenken“ auf vielfachen Ebenen folgt damit tatsächlich dem ihm zu Grunde liegenden Satz aus den Vermischten Bemerkungen aus dem Jahr 1946:

Welch ein kleiner Gedanke doch ein ganzes Leben füllen kann! Wie man doch sein ganzes Leben lang dasselbe kleine Ländchen bereisen kann, und meinen, es gäbe nichts außer ihm! Man sieht alles in einer merkwürdigen Perspektive (oder Projektion): das Land, was man unaufhörlich bereist, kommt einem ungeheuer groß vor; alle umgebenden Länder sieht man wie schmale Randgebiete. Um in die Tiefe zu steigen, braucht man nicht weit reisen; ja, Du brauchst dazu nicht Deine nächste und gewöhnliche Umgebung zu verlassen. (VB 520)

Innerhalb der „gewohnten Umgebung“ „in die Tiefe steigen“ kann eben auch meinen, die eigenen kompositorischen Vorstellungen mit Wittgensteins Sprache, Texten und Ideen neu auszuleuchten. Cage, Zimmermann, Lutyens und Reich tun das auf jeweils ganz eigene Weise und mit höchst unterschiedlichen Ergebnissen.

Ein alle Kompositionen verbindender Reiz, sich auf Wittgenstein einzulassen, liegt aber anscheinend in der Sprache des Philosophen: Wittgensteins Sätze kommen meist ohne komplexen Satzbau aus, die zur Vertonung gewählten weisen oft einen pointierten Stil auf, deren Kürze durch eine gewisse Klanglichkeit unterstrichen wird. Wenn z.B. Numminen den letzten Tractatus-Satz singt, biegt er die satzeigenen Rhythmen für seine Klangidee zurecht: Das erste Wort wird auftaktig, das letzte, im deutschen eigentlich eher weich anmutenden Wort „schweigen“ wird mittels der unnachgiebigen Silbenbetonung der vorangehenden Worte zu einer Art akustischem Faustschlag - der Sinn des Satzes tritt hinter seinem plötzlich ganz neu gehörten Klang völlig zurück.

7 Vergl. den Beitrag von Eggers, „Musik bei Ludwig Wittgenstein“ in diesem Band. 
Die Textauswahl für eine Vertonung oder ein Lied stellt den Komponisten oder die Liedermacherin immer auch vor die Entscheidung, mittels dieser Auswahl gewisse persönliche Eigenschaften oder Gedanken einer Öffentlichkeit preiszugeben. Wittgensteins Sätze sind auf den ersten Blick weder weltanschaulich, noch sentimental oder in Wortschatz oder Bildsprache verbraucht. Gleichzeitig vermitteln Sie aber zumindest für einen von der Wittgensteinforschung nicht zu stark beeinflussten Leser eine gewisse Offenheit der Interpretation und damit das Potential, eigene Kreativität in vielfacher Weise anzuregen und ganz andere „Leitern“ nach dem Aufstieg zurückzulassen, als die etablierten Philosophen: Cage kann Wittgenstein zu einem Material unter vielen machen, Zimmermann braucht nicht nachzuweisen, wo Wittgensteins Gedanken in die seinen übergehen und umgekehrt, und Lutyens reicht gar die Struktur und der Sprachklang. Und so ist es in gewisser Weise bezeichnend, dass Steve Reich, als er sich 2004 in You Are/ Variations wieder Wittgenstein zuwandte, ausgerechnet den Satz vertonte: „Die Erklärungen haben irgendwo ein Ende.“

\section{Literatur}

Birnbacher, Dieter: „Musik und Musikalisches bei Wittgenstein“. In: Musik \& Ästhetik 46, (April 2008), S. 49-64.

Cage, John: I-VI. MethodStructureIntentionDisciplineNotationIndeterminacyInterpenetration ImitationDevotionCircumstancesVariableStructureNonunderstaning ContingencyInconsistency Performance (=The Charles Eliot Norton Lectures, 1988-89), Cambridge/MA 1990.

Eggers, Katrin: Ludwig Wittgenstein als Musikphilosoph (=Musikphilosophie 2), Freiburg i. Br./ München 2011.

Glock, William: „A Tribute to Elisabeth Lutyens“, script of BBC Radio 3 broadcast of 15. December 1983. British Library, Sdd. 71114, f. 220.

Harris, Merion / Harris, Susie: A Pilgrim Soul. The Life and Work of Elisabeth Lutyens. London 1989. Hiekel, Jörn Peter: Bernd Alois Zimmermanns Requiem für einen jungen Dichter (=Beihefte zum Archiv für Musikwissenschaft Bd. XXXVI), Stuttgart 1995.

Lutyens, Elisabeth: A Goldfish Bowl. London 1972.

Lutyens, Elisabeth: Excerpta Tractati logico Philosophici: Motette. op. 27. gemischter Chor (SATB). London: Schott Music Ltd., 1975.

Max, Ingolf: „Familienähnlichkeit als Analysemethode von Spätwerken Beethovens und Wittgensteins“. In: Heinrich, Richard / Nemeth, Elisabeth / Pichler, Wolfram (Hg): Bild und Bildlichkeit in Philosophie, Wissenschaft und Kunst / Image and Imaging in Philosophy, Science, and the Arts. Papers of the 33. International Wittgenstein Symposium, Kirchberg am Wechsel, Austrian Ludwig Wittgenstein Society, Kirchberg am Wechsel 2010, S. 196-200.

Miller, Henry: Tropic of Capricorn. New York 1961.

Perloff, Marjorie: „The music of verbal space: John Cage’s ‘What you say'“. In: Morris, Adalaide (ed.): Sound States: Innovative Poetics and Acoustical Technologies, with accompanying CD, Chapel Hill and London 1997, S. 129-148. 
Reich, Steve: Writings on Music 1965-2000. Edited with an Introduction by Paul Hillier, Oxford 2002.

Stenius, Erik: Wittgensteins Traktat. Eine kritische Darlegung seiner Hauptgedanken. Aus dem Englischen von Wilhelm Bader, Frankfurt/Main 1969.

Tait, Simon: „Mind over Music“. In: The Independent, 12. November 2003.

Woodley, Ronald: „Steve Reich's Proverb, Canon, and a little Wittgenstein“. In: Canons and Canon Techniques, 14th-16th Centuries: Theory, Practice, and Reception History. Proceedings of the International Conference, Leuven, 4.-6. October 2005, Leuven 2007, S. 457-481. 